\title{
Transglutaminase 3 protein modulates human esophageal cancer cell growth by targeting the NF- $\kappa B$ signaling pathway
}

\author{
WEI LI ${ }^{1}$, ZHONGMIAN ZHANG $^{2}$, WENCHAO ZHAO ${ }^{1}$ and NA HAN ${ }^{2}$ \\ ${ }^{1}$ Department of Physiology and Neurobiology, School of Basic Medical Sciences, Zhengzhou University, \\ Zhengzhou, Henan 450001; ${ }^{2}$ Department of Oncology, The Second Affiliated Hospital \\ of Zhengzhou University, Zhengzhou, Henan 450014, P.R. China
}

Received February 29, 2016; Accepted May 13, 2016

DOI: $10.3892 /$ or.2016.4921

\begin{abstract}
It has recently been found that the expression of the transglutaminase 3 (TGM3) is significantly reduced in esophageal cancer (EC). However, the potential of TGM3 as a useful biomarker or as a molecular target for EC diagnosis and treatment is unclear. The aim of the present study was to explore the role of the TGM3 in EC. The expression level of TGM3 were measured by real-time polymerase chain reaction and western blot analysis in EC cell lines, including SKGT-4, KYSE-510, OE33, OE21 and the normal esophageal epithelial cell line HEEC; fifty-eight pairs of tissues samples were also measured. Through exogenous expression of TGM3 in EC cells it was found that the expression of TGM3 is closely associated with tumor cell growth and apoptosis. TGM3 was found downregulated in EC and closely associated with tumor proliferation and migration. In addition, overexpression of TGM3 apparently induces EC cell proliferation, migration, invasion and promotes cell apoptosis in vitro. Subsequent experiments identified the $\mathrm{NF}-\kappa \mathrm{B}$ signaling pathway as direct target of TGM3. Our data collectively demonstrate that TGM3 can be a candidate tumor suppressor that is able to induce EC cell proliferation and migration by downregulating the $\mathrm{NF}-\kappa \mathrm{B}$ signaling pathway, indicating that TGM3 may serve as a useful biomarker and therapeutic target for EC treatment.
\end{abstract}

Correspondence to: Dr Wenchao Zhao, Department of Physiology and Neurobiology, School of Basic Medical Sciences, Zhengzhou University, No. 100 Science Avenue, Zhengzhou, Henan 450001, P.R. China

E-mail: wenchaozhaowcz@sina.com

Dr Na Han, Department of Oncology, The Second Affiliated Hospital of Zhengzhou University, No. 2 Jingba Road, Zhengzhou, Henan 450014, P.R. China

E-mail:nahannh@163.com

Key words: transglutaminase 3, esophageal cancer, tumor suppressor, NF- $\mathrm{BB}$ signaling pathway

\section{Introduction}

Esophageal cancer (EC) is the seventh most common malignant tumor in the world, the mortality of which is ranked sixth among the malignant tumors worldwide (1); its incidence has increased significantly in recent years (2), especially in China. Despite the clinical advances in diagnosis and treatment improvement for malignant tumors, EC remains one of the leading causes of cancer-associated mortality. The overall 5 -year survival rate for all patients with EC is $<20 \%$ (3). Owing to its aggressive nature and poor response to chemotherapy, EC cure is still a problem (4). The incidence and treatment rates of EC will continue to increase substantially with the development of an aging population and the social economic development in China. Therefore, research to identify and develop more effective molecular biomarkers to prevent or treat EC is urgently needed. Further studies have reported that transglutaminase 3 (TGM3) is significantly downregulated in ECs (5-8).

The transglutaminase 3 (TGM 3 ) enzyme is an enzyme with the ability to catalyze the irreversible cross-linking of peptide-bound glutamine residues either with peptidebound lysines or with primary amines (5). It is encoded by the TGM3 gene and widely expressed in the small intestine, brain, skin and mucosa (6). In the skin and mucosa, TGM3 is predominantly expressed in the suprabasal layers of the stratified squamous epithelium $(7,8)$. It has been demonstrated that TGM3 is required for the cross-linking of the structural protein trichohyalin and the keratin intermediate filaments to form a rigid structure within the inner root sheath cells $(9,10)$. Recent studies have revealed that downregulation of the TGM3 gene is closely linked with a variety of human cancer types, including laryngeal carcinoma, esophageal and oral squamous cell carcinoma (OSCC) (11-13). Moreover, Uemura et al (12) identified TGM3 as a novel prognostic indicator in ESCC; the prognostic performance of TGM3 was confirmed by immunohistochemistry in 76 ESCC cases. In addition, Mendez et al (14) reported that the TGM3 gene is differentially expressed in node-positive and node-negative primary tumors in patients with OSCC, implying that decreased TGM 3 expression might contribute to the metastatic potential of OSCC. However, the biological function and molecular mechanism of the TGM3 gene in cancer initiation and progression have 
not been reported. In addition, whether the TGM3 gene might be a valuable diagnostic or therapeutic biomarker for cancer, especially for EC, needs to be further investigated.

The nuclear factor kappa $\mathrm{B}(\mathrm{NF}-\kappa \mathrm{B})$ transcription factor family is composed of p50, p52, RelA/p65, c-rel and Rel B. The homodimers and heterodimers of these molecules are sequestered in the cytoplasm in an inactive form by the inhibitor of kappa B (I $\mathrm{B})$. Upon stimulation, the $\mathrm{I} \kappa \mathrm{B}$ kinase complex (IKK) phosphorylates the $\kappa \mathrm{B}$ inhibitor, which then releases $\mathrm{NF}-\kappa \mathrm{B}$ and allows its phosphorylation, nuclear translocation, binding and subsequent activation of target genes involved in the regulation of cell proliferation, survival, angiogenesis and metastasis (15). Constitutively active NF- $\kappa \mathrm{B}$ is common in human cancer cell lines as well as tumor tissues derived from patients, but is rare in normal cells (16). In some types of human cancer, there is strong evidence of $N F-\kappa B$ being involved in cancer progression (17), thus, making NF- $\kappa \mathrm{B}$ and its downstream signals promising targets for therapeutic intervention. However, the role of the NF- $\mathrm{NB}$ signaling pathway in the tumorigenesis of human EC is not fully understood.

In the present study, we confirmed that the expression levels of TGM3 are downregulated in EC cell lines compared with normal primary esophageal epithelial cells, and tissue specimens compared with paired adjacent normal tissues, by means of RT-PCR and western blotting. We further evaluated the effect of ectopic TGM3 expression in the SKGT-4, KYSE-510, OE33 and OE21 cell lines. We provide the first evidence that the ectopic expression of TGM3 in EC cell lines inhibits cell proliferation and migration and induces apoptosis in cancer cells in vitro. In addition, we demonstrate that the $\mathrm{NF}-\kappa \mathrm{B}$ signaling pathway is a direct target of TGM3 and show that TGM3 functions as an oncogene by activating the $\mathrm{NF}-\kappa \mathrm{B}$ signaling pathway. These results suggest that TGM3 might be a candidate tumor suppressor contributing to EC and could act as a valuable prognostic predictor for patients with EC.

\section{Materials and methods}

Tissue samples. Fifty-eight pairs of primary EC tissue samples and adjacent non-cancerous tissues (located $>3 \mathrm{~cm}$ away from the tumor) were obtained from the Department of Oncology, The Second Affiliated Hospital, Zhengzhou University (Zhengzhou, China) between June 2014 and December 2015. All subjects were diagnosed and confirmed by a pathological evaluation. None of the subjects received any biotherapy or chemotherapy treatment before recruitment to this study. The study was approved by the Institutional Ethics Committee of the Second Affiliated Hospital, Zhengzhou University and all of the patients provided written informed consent in accordance with the institutional guidelines.

Cell culture and transfection. The EC cell lines SKGT-4 and KYSE-510 were purchased from the American Type Culture Collection (ATCC; Manassas, VA, USA). OE33 and OE21 were obtained from the European Collection of Animal Cell Cultures (ECACC; Porton Down, Salisbury, UK). All EC cell lines were grown in RPMI-1640 medium supplemented with $10 \%$ filtered fetal calf serum, 100 units $/ \mathrm{ml}$ penicillin, $100 \mu \mathrm{g} / \mathrm{ml}$ streptomycin and $2 \mathrm{mM}$ L-glutamine. In addition, the normal human esophageal epithelial cell line HEEC was grown in Dulbecco's modified Eagle's medium (DMEM) with $10 \%$ FBS. All cells were cultured in a humidified atmosphere of $5 \% \mathrm{CO}_{2}$ at $37^{\circ} \mathrm{C}$.

Bay11-7082, a specific inhibitor of NF- $\kappa \mathrm{B}$ signaling, was purchased from CsA (Biomol, Plymouth Meeting, PA) and diluted in culture medium to obtain the desired concentration. Recombinant human TGM3 was obtained from Abcam Inc. (Cambridge, MA, USA), and the rabbit anti-TGM3 antibody was from Adipo Bioscience Inc. (Santa Clara, CA, USA). EC cells were transfected with recombinant human TGM3 $(10 \mu \mathrm{g})$ or TGM3 antibody (1:400; Neomarkers, Fremond, CA, USA) with Lipofectamine 2000 (Invitrogen, Carlsbad, CA, USA) according to the manufacturer's instructions; EC cells without any treatment were used as controls.

RNA isolation and quantitative real-time PCR ( $q R T-P C R)$. Total RNA was isolated using the TRIzol reagent (Invitrogen Life Technologies). The concentration of RNA were determined using a NanoDrop ND-1000 instrument (Thermo Fisher Scientific, Waltham, MA, USA), and aliquots of the samples were stored at $-80^{\circ} \mathrm{C}$. For reverse transcription, cDNA was synthesized by using a reverse transcription kit (Promega, Madison, WI, USA). Quantitative real-time polymerase chain reaction (qRT-PCR) analysis was performed (StepOne; Applied Biosystems, Foster City, CA, USA) using SYBRGreen (Takara, Shiga, Japan). All PCR experiments were performed in triplicate. The TGM3 primer sequences are as follows: sense: 5'-TCAACTGGCAGACGGCCTTCA-3' and antisense 5'-GTACCGTCCTATGGGTGCGCT-3'.

Western blot analysis. Total protein was extracted using RIPA lysis buffer and the concentrations in different samples were determined using the BCA kit (Beyotime Institute of Biotechnology, Haimen, China). An equivalent amount of protein $(30 \mu \mathrm{g})$ from different samples was separated by SDS-PAGE, and then transferred onto a nitrocellulose membrane (Millipore, Bedford, MA, USA). The membrane was blocked with $3.0 \%$ non-fat milk at room temperature for $1 \mathrm{~h}$ and then blotted with rabbit anti-TGM3 antibody or mouse anti- $\beta$-actin antibody $(1: 10,000)$ purchased from Sigma (San Francisco, CA, USA) overnight at $4^{\circ} \mathrm{C}$. After incubation with horseradish peroxidase-conjugated secondary antibodies (1:2,000; Bioss, Beijing, China) for $1 \mathrm{~h}$ at room temperature, the protein complexes was detected using enhanced chemiluminescence reagents (Pierce, Rockford, IL, USA). $\beta$-actin was used as the internal control. The signal intensity on the scanned images was analyzed using Image-Pro Plus 6.0 software.

Cell proliferation assay. Cells were seeded in 96-well plates at $6 \times 10^{3}$ cells/well and the surviving fractions were determined at $0,24,48,72$ and $96 \mathrm{~h}$ using the MTT assay, as previously described (18). The absorbance of each well was measured with a spectrophotometer (Bio-Rad Laboratories, Hercules, CA, USA) at $570 \mathrm{~nm}$. Each experiment was performed in triplicate (19).

Cell migration and invasion assays. EC cells were grown to confluence in 12-well plastic dishes and treated with recombinant human TGM3 protein or a scramble peptide. Then, $24 \mathrm{~h}$ after transfection, linear scratch wounds (in triplicate) were 

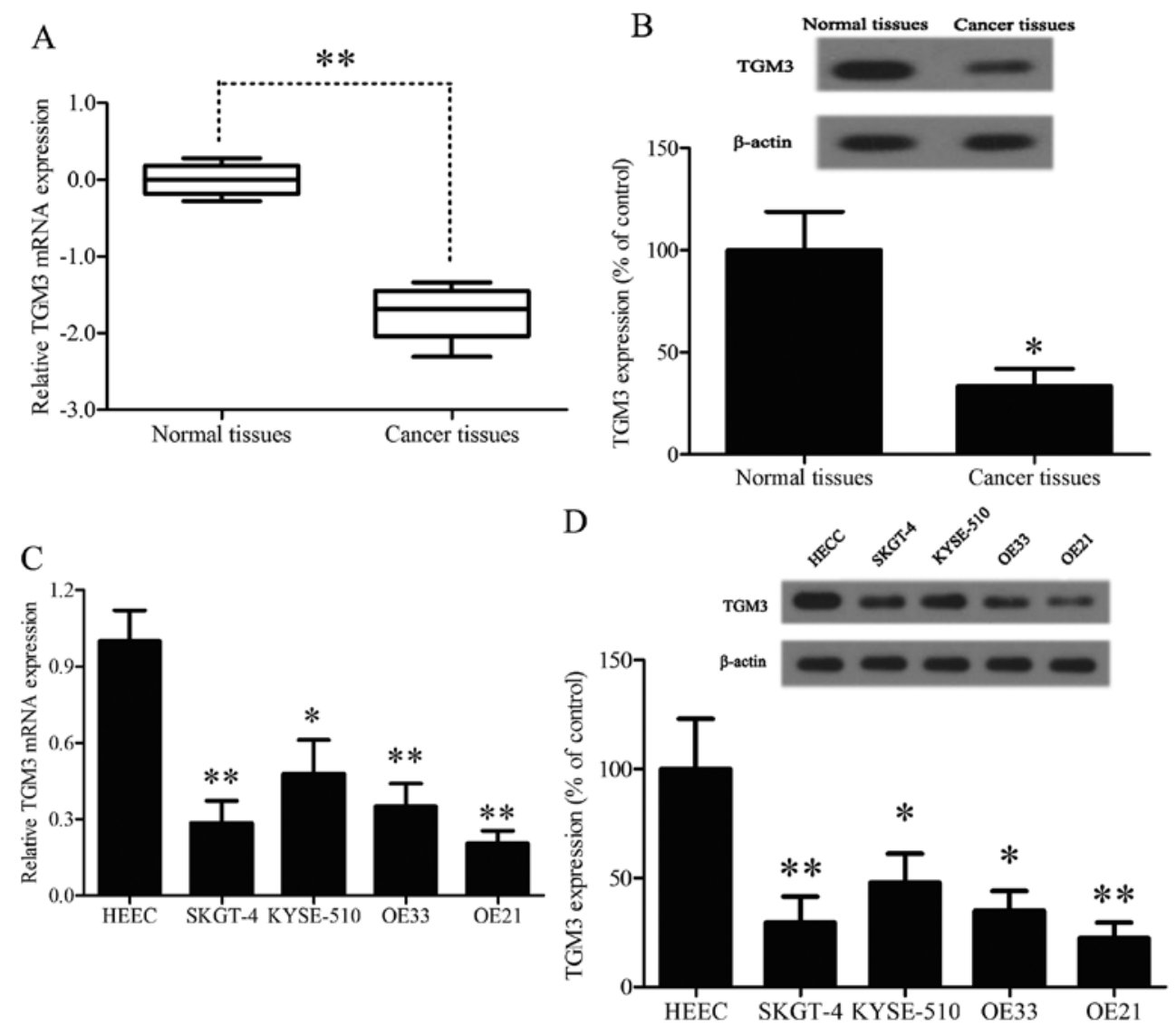

Figure 1. TGM3 expression is frequently downregulated in EC tissues and cell lines. The expression of TGM3 in EC tissues and their matched adjacent noncancerous tissues was determined using (A) RT-PCR and (B) western blot analysis. The expression level of TGM3 in four EC cell lines and the normal human esophageal epithelial cell line HEEC were assessed by (C) RT-PCR and (D) western blot analysis. Data are representative of three experiments. Error bars represent mean $\pm \mathrm{SD}$. ${ }^{*} \mathrm{P}<0.05$ and ${ }^{* *} \mathrm{P}<0.01$, differences from their respective controls, respectively.

created on the confluent cell monolayers using a $200 \mu$ pipette tip. To remove cells from the cell cycle prior to wounding, cells were maintained in serum-free media. A total of 10 areas were selected randomly from each well, and the cells in three wells from each group were quantified.

For the invasion assays, $24 \mathrm{~h}$ after transfection, $1 \times 10^{5}$ cells in serum-free media were seeded in Transwell migration chambers. The upper chamber of the Transwell inserts was coated with Matrigel (Sigma-Aldrich, St. Louis, MO, USA). Medium containing 20\% FBS was added to the lower chamber. After $24 \mathrm{~h}$, the non-invading cells were removed with cotton wool. Invasive cells located on the lower surface of the chamber were stained with May-Grunwald-Giemsa stain (Sigma-Aldrich) and counted using a microscope (Olympus Corp., Tokyo, Japan). From these images, the number of invasive cells was counted. Experiments were independently repeated three times.

Apoptosis assay. Apoptosis was assayed by an Annexin V apoptosis detection kit (BD Biosciences, San Diego, CA, USA). Following transfection for $72 \mathrm{~h}$, the cells were collected and detected using an Annexin V fluorescein isothiocyanate kit (FITC) according to the manufacturer's instructions. In brief, cells transfected with the recombinant human vasostatin-2 or vasostatin- 2 antibody were resuspended in $100 \mathrm{ml}$ binding buffer, at a density of $1 \times 10^{6}$ cells $/ \mathrm{ml}$, then incubated with Annexin V-FITC and PI for $15 \mathrm{~min}$. The cells were analyzed with Beckman CXP software on a FC-500 flow cytometer (Beckman Coulter, Pasadena, CA, USA) within $1 \mathrm{~h}$ of cell collection.

ELISA analysis. The levels of p65 concentrations in culture medium were determined by ELISA (Tanjin Biotechnology Co., Shanghai, China) following the manufacturer's instructions. The absorbance was assessed at $450 \mathrm{~nm}$ using a $680 \mathrm{XR}$ microplate reader (Bio-Rad Laboratories).

Statistical analysis. The differences were analyzed using Student's t-test for two groups and examined by the $\chi^{2}$ test. A $\mathrm{P}$-value $<0.05$ was considered to indicate statistically significant differences. Data were processed as mean \pm standard deviation (SD). All statistical analyses were performed using SPSS 16.0 software (SPSS, Inc., Chicago, IL, USA).

\section{Results}

TGM3 is downregulated in EC tissues and cell lines. In order to confirm the potential roles of TGM3 in EC, RT-PCR was performed to investigate the mRNA levels of TGM3 in 58 paired EC specimens compared to the corresponding adjacent non-cancerous tissues (NCTs) (Fig. 1A). The TGM3 mRNA levels in the EC specimens were significantly lower than those of non-cancerous tissues. Western blot assay was performed to investigate the protein levels of TGM3 in EC specimens, 

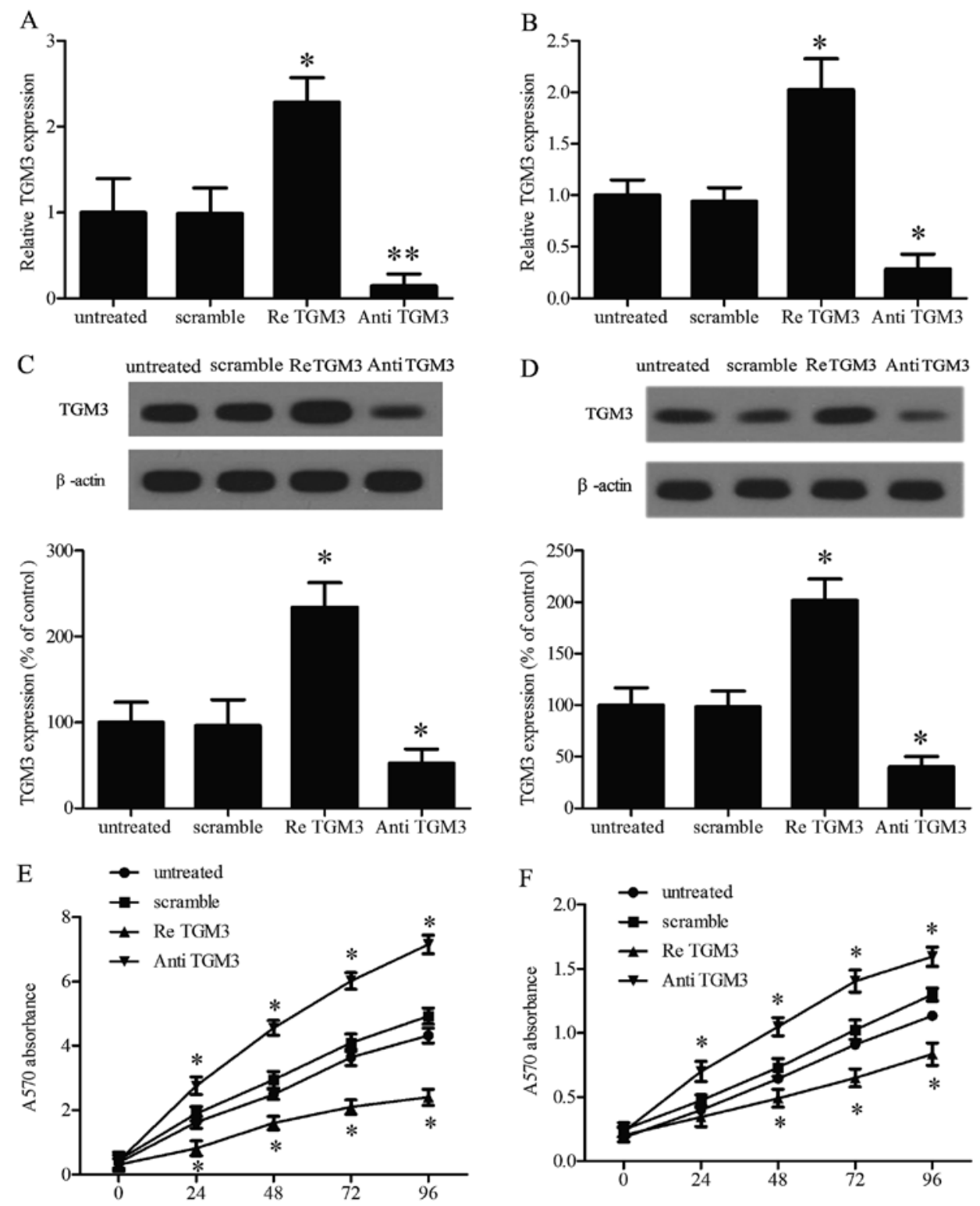

Figure 2. TGM3 protein affects EC cell proliferation in vitro. The relative expression of TGM3 in SKGT-4 cell and KYSE-510 cell lines transfected with recombinant human TGM3 (Re TGM3) or TGM3 antibody (Anti-TGM3) compared with the control (Scramble TGM3) was determined by qRT-PCR (A and B) and western blot analysis (C and D). Proliferation rates of the SKGT-4 and KYSE-510 cell lines were assessed by the MTT assay (E and F). "P<0.05 and ${ }^{* *} \mathrm{P}<0.01$, differences from their respective controls, respectively.

same as above, the protein levels of TGM3 were significantly downregulated in the EC specimens compared to the corresponding adjacent non-cancerous tissues (Fig. 1B). In addition, RT-PCR and western blot assay were performed to investigate the expression level of four EC cell lines and a normal human esophageal epithelial cell line. The TGM3 mRNA levels in the EC cell lines were significantly lower than those of normal esophageal epithelial cells (Fig. 1C). Correspondingly, the protein levels of TGM3 were markedly downregulated in the four EC cell lines compared with the levels in normal epithelial cells using western blot analysis (Fig. 1D). These data show that the expression of TGM3 is universally downregulated in EC cells and patients, indicating that a change of TGM3 expression may affect the development of EC.

Ectopic expression of TGM3 directly affects cell proliferation in vitro. It is well-known that cell proliferation is a key event in the formation and development of cellular oncogenesis. In order to investigate the role of TGM3 in EC cell proliferation, TGM3 was overexpressed or repressed in SKGT-4 cells and KYSE-510 cells by transfection with recombinant human TGM3 or TGM3 antibody, qRT-PCR (Fig. 2A and B) and western blot analysis (Fig. 2C and D) evaluation demonstrated TGM3 expression in SKGT-4 and KYSE-510 cell lines. The MTT assay showed that overexpression of TGM3 inhibited cell proliferation. Conversely, downregulating the expression of TGM3 accelerated cell proliferation in comparison with the control group (Fig. 2E and F). Similar data were also obtained in the other two cell lines (data not shown).

Ectopic expression of TGM3 protein affects cell apoptosis in EC cells. One of the hallmarks of cancer is its ability to evade apoptosis (20). To investigate the effect of TGM3 on EC cell apoptosis, we performed a series of experiments in EC cell 


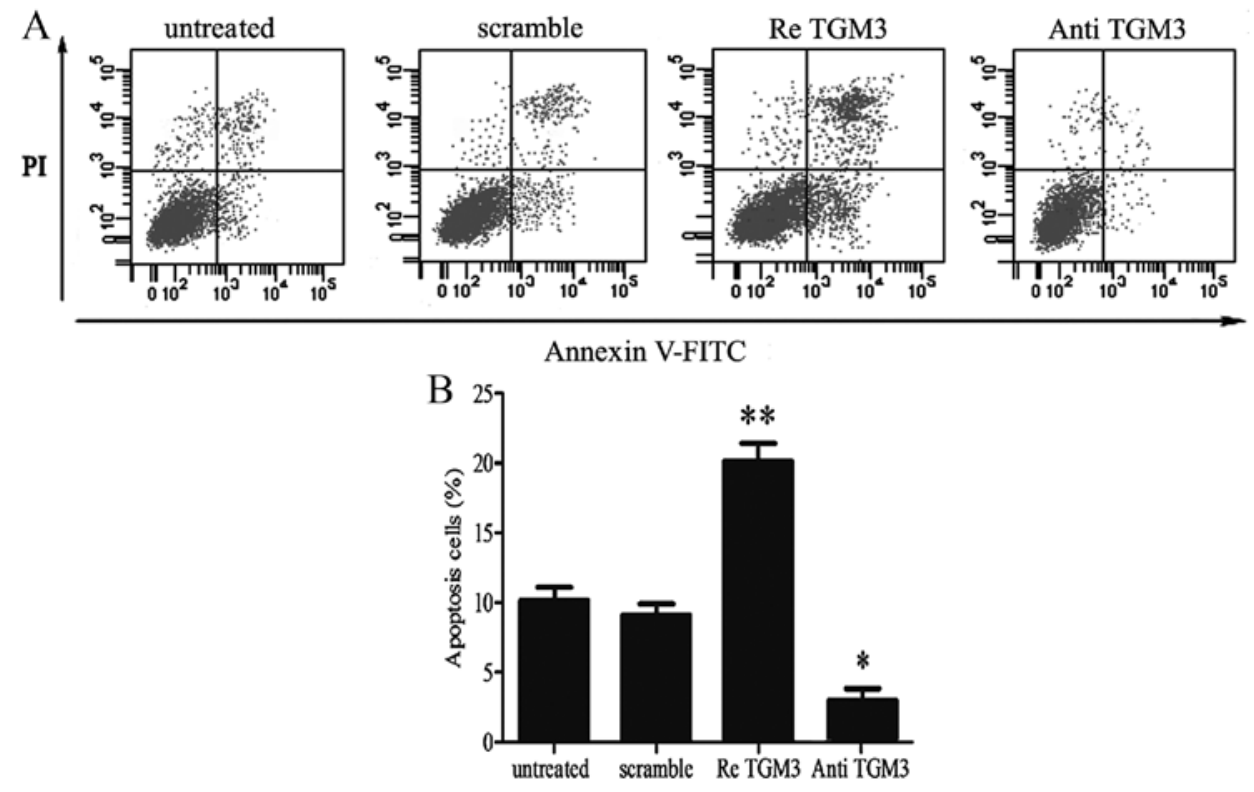

Figure 3. TGM3 affects EC cells apoptosis in vitro. (A) The effect on EC cell apoptosis rate was analyzed after staining with Annexin V-FITC and PI staining. (B) Quantification of apoptosis in SKGT-4 cells transfected with transfection with recombinant human TGM3 or TGM3 antibody for $48 \mathrm{~h}$. Data represent means \pm SD from three independent experiments. ${ }^{*} \mathrm{P}<0.05^{* *} \mathrm{P}<0.01$, differences from their respective controls, respectively.
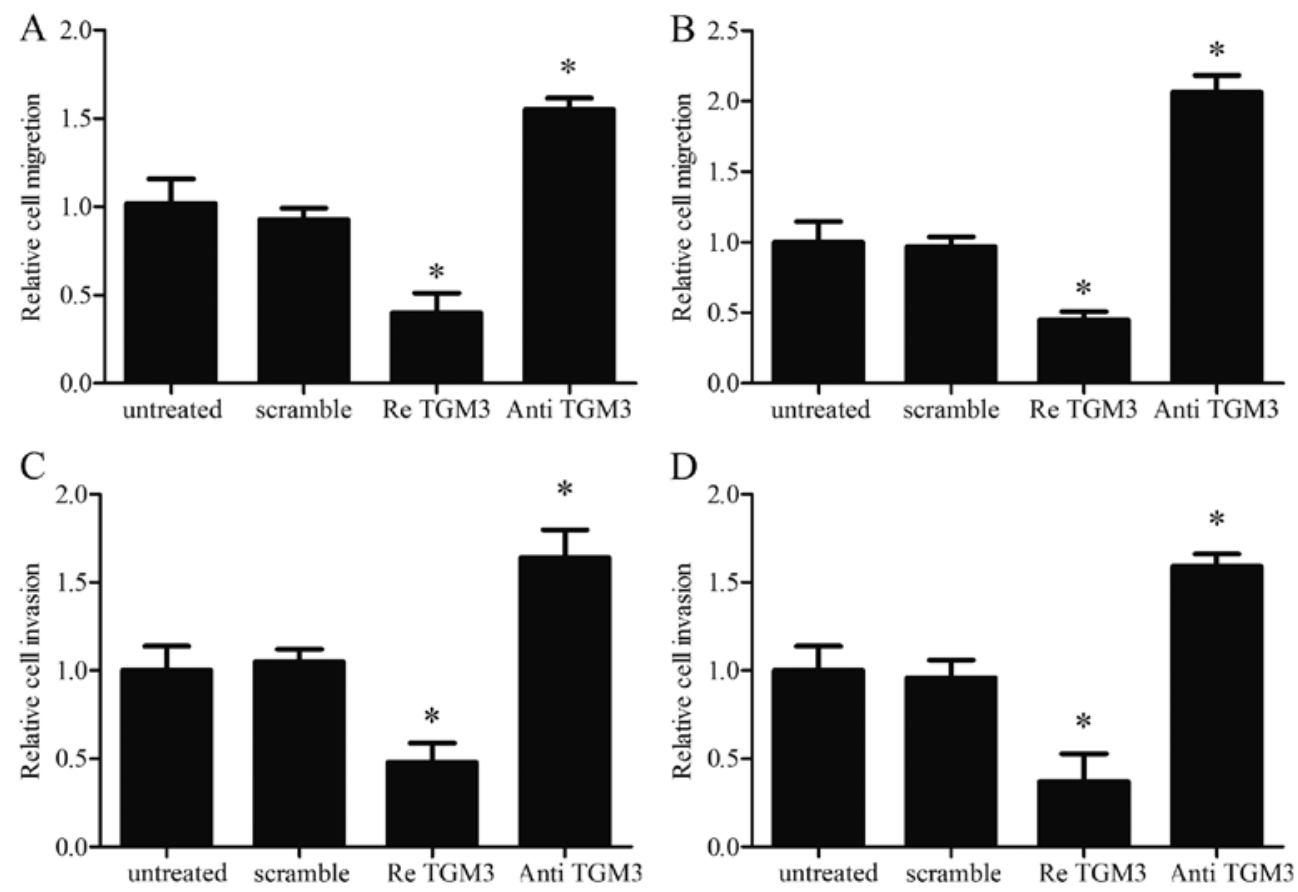

Figure 4. TGM3 protein affects EC cell migration and invasion in vitro. Scratch assays were performed to analyze the effect of TGM3 on the migration of (A) SKGT-4 cells and (B) KYSE-510 cells. Transwell assays were used to analyze the effect of TGM3 on the invasion of (C) SKGT-4 cells and (D) KYSE-510 cells. ${ }^{*} \mathrm{P}<0.05$, differences from the respective control.

lines, which were stimulated by transfection with recombinant human TGM3 or TGM3 antibody to obtain TGM3 protein ectopic expression. We then investigated the effect of TGM3 on EC cell apoptosis using Annexin V-FITC staining. As shown in Fig. 3A, overexpression of TGM3 significantly increased apoptosis in SKGT-4 cells. The percentage of apoptotic cells increased to $9.8 \%$ after transfection with recombinant human TGM3. Suppressed expression of TGM3 increased apoptosis in SKGT-4 cells (Fig. 3B). Overall, these data indicate that TGM3 may play the role of promoting apoptosis in EC cells.

Ectopic expression of TGM3 directly affects cell migration and invasion in vitro. To analyze the role of TGM3 in cell migration and invasion, which are the key determinants of malignant progression and metastasis, scratch assays and Transwell assays were performed in the SKGT-4 and KYSE-510 cell lines. Cells treated with recombinant human TGM3 were distinctively less 

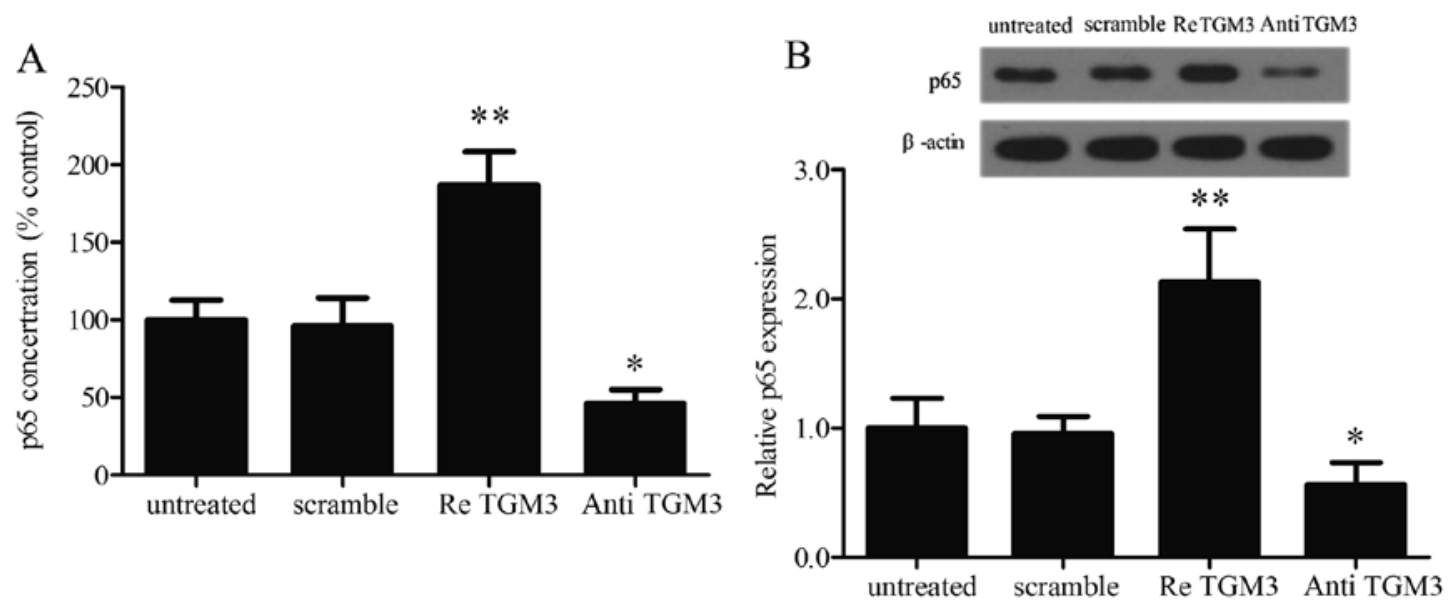

Figure 5. Overexpression of TGM3 triggered activation of the NF- $\mathrm{kB}$ signaling pathway. (A) After pretreatment with recombinant human TGM3 (Re TGM3) or TGM3 antibody (Anti TGM3), the p65 concentration in the culture medium of SKGT-4 cells was assessed by ELISA. (B) The protein expression of p65 was analyzed by western blotting. Also, the $\mathrm{p} 65$ concentration and protein expression in SKGT- 4 cells were analyzed. Data represent means \pm SD from three independent experiments. ${ }^{*} \mathrm{P}<0.05,{ }^{* *} \mathrm{P}<0.01$ differences from their respective controls, respectively.
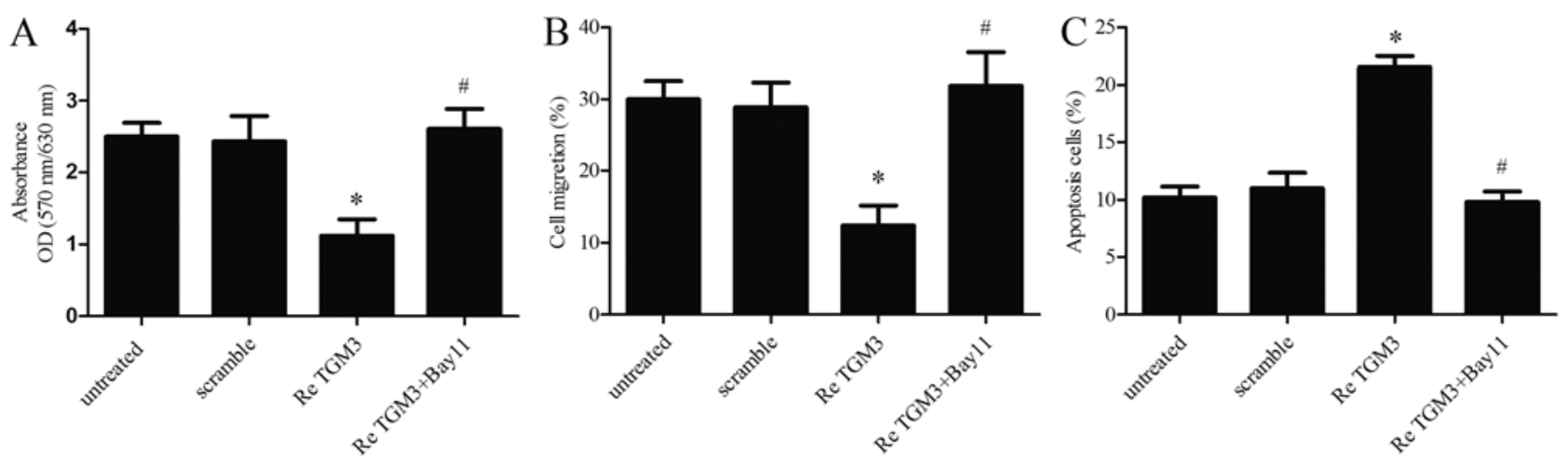

Figure 6. TGM3 regulates EC cell development by targeting NF-kB signaling pathway. (A) The MTT assay shows that overexpression of TGM3 increases the effect of TGM3 in the regulation of proliferation. (B) Inhibition of the NF-KB signaling pathway rescued cell migration induced by TGM3 in EC cells. (C) Disruption of the NF-kB signaling pathway promoted apoptosis induced by TGM3 in EC cells. Data represent mean \pm SD. ${ }^{*} \mathrm{P}<0.01$, differences vs. the normal control; ${ }^{*} \mathrm{P}<0.01$, differences vs. the TGM3 scramble.

migratory and invasive; however, cells treated with the TGM3 antibody were distinctively more migratory and invasive than untreated cells at $48 \mathrm{~h}$ after scratching (Fig. 4). These results indicate that TGM3 plays an important role in regulating the proliferation of EC cells and strongly suggest that introduction of TGM3 could inhibit cellular oncogenesis by suppressing the migration and invasion of EC cells.

Overexpression of TGM3 promotes activation of the $N F-\kappa B$ signaling pathway. The NF- $\mathrm{KB}$ signaling pathway has been demonstrated to play an important role in cellular energy homeostasis and reported to be extensively involved in cell proliferation, migration and differentiation (21-23). To further explore the mechanisms involved in TGM3 induced tumor cell growth and apoptosis, we analyzed the NF- $\mathrm{KB}$ pathway at the protein level via ELISA and western blotting. The results show that elevated p65 concentrations in culture medium of SKGT-4 cells were found with TGM3 overexpression (Fig. 5A). Furthermore, the protein expression of p65 was also found to be markedly increased after TGM3 pre-treatment (Fig. 5B); the same effect was observed in the other three cell lines. These results suggest that TGM3 induces activation of the NF-кB pathway.

TGM3 modulates EC cell growth and apoptosis by targeting the $N F-\kappa B$ signaling pathway. To further delineate the effect of NF- $\kappa B$ on TGM3 modulated EC cell growth and apoptosis, the NF- $\mathrm{KB}$ signaling inhibitor Bay11-7082 (20 mol/l) was used. After a series of functional restoration assays, we found that the decrease in SKGT-4 cell proliferation (Fig. 6A) and migration (Fig. 6B) induced by TGM3 upregulation was markedly strengthened following Bay11-7082 stimulation. Moreover, disruption of NF- $\kappa \mathrm{B}$ signaling by Bay11-7082 treatment also significantly decreased the apoptosis rate (Fig. 6C). These results indicate that TGM3 may repress EC cell proliferation and migration and promote apoptosis by modulating the NF- $\mathrm{KB}$ signaling pathway.

\section{Discussion}

A variety of treatments is currently available for EC. The choice of treatment is crucial, recurrent or distant metastases 
as the limitations of conventional treatments, therefore, it is necessary to search for novel approaches. Recently, several research groups have reported certain genes and signaling molecules that are potentially involved in EC initiation and progression $(24,25)$. These molecular targets may provide significant clues for early diagnosis, prognosis and new targeted therapies (26-28). Previous studies have found that TGM3 expression is downregulation in head and neck cancer, laryngeal carcinoma and oral squamous cell carcinoma (29-32), other reports found that TGM3 expression is decreased in EC tissues compared with normal tissues (29). However, the exact function and mechanisms of TGM3 in EC are largely unknown. In this study, we verified this finding by assessing the mRNA and protein levels of TGM3 in four EC cell lines and 58 EC specimens. We found that the levels of this TGM3 were much lower in EC tissues and cell lines compared with adjacent controls. Our findings are consistent with previous reports, these results allowed us to speculate that the overexpression of TGM3 may confer a survival advantage to EC patients. Since metastasis is the major cause of morbidity and mortality in EC patients, we investigated the roles of TGM3 in cell migration and invasion of EC cell lines. Transglutaminase (TGM) enzymes are widespread in both plants and animals, they are a family of calcium-dependent enzymes that catalyze the formation of isopeptide bonds $(34,35)$. TGM3 is expressed predominantly during the late stages of the terminal differentiation of the epidermis and in certain cell types of hair follicles (36). Although TGM3 downregulation has been found in many cases, however, little information is available concerning its function and mechanisms involvement in EC. The results of this study indicate that TGM3 plays an important role in regulating the proliferation of EC cells and strongly suggest that the introduction of TGM3 could inhibit cellular oncogenesis by suppressing the migration and invasion of EC cells. We consider TGM3 to be a strong biomarker candidate of EC, because its expression clearly correlated with cancer progression in our study and it has also been shown to be potentially relevant to ESCC (esophageal squamous cell carcinoma) (37).

Further studies identified that TGM3 promotes activation of the NF- $\kappa \mathrm{B}$ signaling pathway. Recently, abundant studies have confirmed the important roles of NF- $\kappa \mathrm{B}$ signaling in cancer development and progression, including proliferation, survival, angiogenesis and metastasis (38). The significance of $\mathrm{NF}-\kappa \mathrm{B}$ and its therapeutic value as a target for cancer therapy have been investigated in several types of human cancer $(39,40)$. Only a handful of studies to date have explored the involvement of $\mathrm{NF}-\kappa \mathrm{B}$ activation in $\mathrm{EC}$ (41-43) in which the $\mathrm{NF}-\kappa \mathrm{B}$ status correlated positively with metastasis, resistance to chemotherapy and patient survival (44-46). The present study broadens our understanding of the roles that TGM3 plays in EC by targeting $\mathrm{NF}-\kappa \mathrm{B}$ signaling and thus indicates that it can be a powerful target for cancer therapy.

In conclusion, this study showed that TGM3 expression is low in EC tissues and cell lines, this result is consistent with the previous studies. We also found that in EC cell lines overexpression of TGM3 inhibits cell proliferation and induces apoptosis by activation of $\mathrm{NF}-\kappa \mathrm{B}$ signaling pathway. As the TGM3 expression level is associated with EC cell growth process, promotion of TGM3 expression in EC tissues may inhibit tumor cell amplification. Therefore, the present study may provide a therapeutic strategy for patients with EC.

\section{Acknowledgements}

The present study is supported by grants from the Key Scientific Research Project of Henan Province (14A310017) and the Key Project of Henan Provincial Department of Science and Technology (142102310311).

\section{References}

1. Kamangar F1, Qiao YL, Schiller JT, Dawsey SM, Fears T, Sun XD, Abnet CC, Zhao P, Taylor PR and Mark SD: Human papillomavirus serology and the risk of esophageal and gastric cancers: results from a cohort in a high-risk region in China. Int J Cancer 119: 579-584, 2006.

2. Jemal A, Bray F, Center MM, Ferlay J, Ward E and Forman D: Global cancer statistics. CA Cancer J Clin 61: 69-90, 2011.

3. Siegel R, Naishadham D and Jemal A: Cancer statistics for Hispanics/Latinos, 2012. CA Cancer J Clin 62: 283-298, 2012.

4. Gaur P, Kim MP and Dunkin BJ: Esophageal cancer: Recent advances in screening, targeted therapy, and management. J Carcinog 13: 11, 2014.

5. Ahvazi B, Kim HC, Kee SH, Nemes Z and Steinert PM: Threedimensional structure of the human transglutaminase 3 enzyme: Binding of calcium ions changes structure for activation. EMBO J 21: 2055-2067, 2002.

6. Hitomi K, Horio Y, Ikura K, Yamanishi K and Maki M: Analysis of epidermal-type transglutaminase (TGase 3) expression in mouse tissues and cell lines. Int J Biochem Cell Biol 33: 491-498, 2001.

7. Hitomi K: Transglutaminases in skin epidermis. Eur J Dermatol 15: 313-319, 2005

8. Hitomi K, Presland RB, Nakayama T, Fleckman P, Dale BA and Maki M: Analysis of epidermal-type transglutaminase (transglutaminase 3) in human stratified epithelia and cultured keratinocytes using monoclonal antibodies. J Dermatol Sci 32: 95-103, 2003

9. Kalinin AE, Kajava AV and Steinert PM: Epithelial barrier function: Assembly and structural features of the cornified cell envelope. BioEssays 24: 789-800, 2002.

10. Eckert RL, Sturniolo MT, Ann-Marie B, Monica R and Rorke EA: Transglutaminase function in epidermis. JJ Invest Dermatol 124: 481-492, 2005.

11. Guang HE, Zhao Z, Weineng FU, Sun X, Zhenming XU and Sun K: Study on the loss of heterozygosity and expression of transglutaminase 3 gene in laryngeal carcinoma. Zhonghua $\mathrm{Yi}$ Xue Yi Chuan Xue Za Zhi 19: 120-123, 2002 (In Chinese).

12. Uemura N, Nakanishi Y, H, Saito S, Nagino M, Hirohashi S and Kondo T: Transglutaminase 3 as a prognostic biomarker in esophageal cancer revealed by proteomics. Int J Cancer 124: 2106-1255, 2009

13. Negishi A, Masuda M, Ono M, Honda K, Shitashige M, Satow R, Sakuma T, Kuwabara H, Nakanishi Y, Kanai Y, et al: Quantitative proteomics using formalin-fixed paraffin-embedded tissues of oral squamous cell carcinoma. Cancer Sci 100: 1605-1611, 2009.

14. Méndez E, Fan W, Choi P, Agoff SN, Whipple M, Farwell DG, Futran ND, Weymuller EA Jr, Zhao LP and Chen Cl: Tumorspecific genetic expression profile of metastatic oral squamous cell carcinoma. Head Neck 29: 803-814, 2007.

15. Viatour P, Merville MP, Bours V and Chariot A: Phosphorylation of NF-kappaB and IkappaB proteins: Implications in cancer and inflammation. Trends Biochem Sci 30: 43-52, 2005.

16. Li B, Li YY, Tsao SW and Cheung AL: Targeting NF-kappaB signaling pathway suppresses tumor growth, angiogenesis, and metastasis of human esophageal cancer. Mol Cancer Ther 8: 2635-2644, 2009.

17. Bassères DS and Baldwin AS: Nuclear factor-kappaB and inhibitor of kappaB kinase pathways in oncogenic initiation and progression. Oncogene 25: 6817-6830, 2006.

18. Bartel DP: MicroRNAs: Genomics, biogenesis, mechanism, and function. Cell 116: 281-297, 2004.

19. Zhang L, Liu X, Jin H, Guo X, Xia L, Chen Z, Bai M, Liu J, Shang $\mathrm{X}, \mathrm{Wu} \mathrm{K}$, et al: miR-206 inhibits gastric cancer proliferation in part by repressing cyclinD2. Cancer Lett 332: 94-101, 2013. 
20. Cotter TG: Apoptosis and cancer: The genesis of a research field. Nat Rev Cancer 9: 501-507, 2009.

21. Darius W, Ilja M, Margitta E, Christian K and Barbara K: Tumor necrosis factor alpha triggers proliferation of adult neural stem cells via IKK/NF-kappaB signaling. BMC Neurosci 7: 1-18, 2006.

22. Palumbo R, Galvez BG, Pusterla T, De Marchis F, Cossu G, Marcu KB and Bianchi ME: Cells migrating to sites of tissue damage in response to the danger signal HMGB1 require NF-kappaB activation. J Cell Biol 179: 33-40, 2007.

23. Wu Y and Zhou BP: TNF-alpha/NF-kappaB/Snail pathway in cancer cell migration and invasion. Br J Cancer 102: 639-644, 2010.

24. Wong GS, Lee JS, Park YY, Klein-Szanto AJ, Waldron TJ, Cukierman E, Herlyn M, Gimotty P, Nakagawa $\mathrm{H}$ and Rustgi AK Periostin cooperates with mutant $\mathrm{p} 53$ to mediate invasion through the induction of STAT1 signaling in the esophageal tumor microenvironment. Oncogenesis 2: e59, 2013.

25. Kaz AM, Luo Y, Dzieciatkowski S, Chak A, Willis JE, Upton MP, Leidner RS and Grady WM: Aberrantly methylated PKP1 in the progression of Barrett's esophagus to esophageal adenocarcinoma. Genes Chromosomes Cancer 51: 384-393, 2012.

26. Shigaki H, Baba Y, Watanabe M, Murata A, Ishimoto $T$, Iwatsuki M, Iwagami S, Nosho K and Baba H: PIK3CA mutation is associated with a favorable prognosis among patients with curatively resected esophageal squamous cell carcinoma. Clin Cancer Res 19: 2451-2459, 2013.

27. Shah AK, Cao KA, Choi E, Chen D, Gautier B, Nancarrow D, Whiteman DC, Saunders NA, Barbour AP, Joshi V, et al: Serum glycoprotein biomarker discovery and qualification pipeline reveals novel diagnostic biomarker candidates for esophageal adenocarcinoma. Mol Cell Proteomics 14: 3023-3039, 2015.

28. Isozaki Y, Hoshino I, Nohata N, Kinoshita T, Akutsu Y, Hanari N, Mori M, Yoneyama Y, Akanuma N, Takeshita N, et al: Identification of novel molecular targets regulated by tumor suppressive miR-375 induced by histone acetylation in esophageal squamous cell carcinoma. Int J Oncol 41: 985-994, 2012.

29. Uemura N, Nakanishi Y, Kato H, Saito S, Nagino M, Hirohashi S and Kondo T: Transglutaminase 3 as a prognostic biomarker in esophageal cancer revealed by proteomics. Int J Cancer 124: 2106-2115, 2009.

30. Wu X, Cao W, Wang X, Zhang J, Lv Z, Qin X, Wu Y and Chen W: TGM3, a candidate tumor suppressor gene, contributes to human head and neck cancer. Mol Cancer 12: 151, 2013.

31. Liu J, Zhou Y, Wan J and Liu Z: Expression of TGM3 protein and its significance in laryngeal carcinoma. Lin Chung Er Bi Yan Hou Tou Jing Wai Ke Za Zhi 26: 101-103, 2012 (In Chinese).

32. Ye H, Yu T, Temam S, Ziober BL, Wang J, Schwartz JL, Mao L, Wong DT and Zhou X: Transcriptomic dissection of tongue squamous cell carcinoma. BMC Genomics 9: 69, 2008.

33. Kondoh N, Ishikawa T, Ohkura S, Arai M, Hada A, Yamazaki Y, et al: Gene expression signatures that classify the mode of invasion of primary oral squamous cell carcinomas. Mol Carcinog 47: 744-756, 2008.
34. Lorand L and Graham RM: Transglutaminases: Crosslinking enzymes with pleiotropic functions. Nat Rev Mol Cell Biol 4: 140-156, 2003.

35. Griffin M, Casadio R and Bergamini CM: Transglutaminases: Nature's biological glues. Biochem J 368: 377-396, 2002.

36. Candi E, Schmidt R and Melino G: The cornified envelope: A model of cell death in the skin. Nat Rev Mol Cell Biol 6: 328-340, 2005.

37. Liu W, Yu ZC, Cao WF, Ding F and Liu ZH: Functional studies of a novel oncogene TGM3 in human esophageal squamous cell carcinoma. World J Gastroenterol 12: 3929-3932, 2006.

38. Karin $M$ and Greten FR: NF-kappaB: Linking inflammation and immunity to cancer development and progression. Nat Rev Immunol 5: 749-759, 2005.

39. Sourbier C, Danilin S, Lindner V, Steger J, Rothhut S, Meyer N, Jacqmin D, Helwig JJ, Lang H and Massfelder T: Targeting the nuclear factor-kappaB rescue pathway has promising future in human renal cell carcinoma therapy. Cancer Res 67: 11668-11676, 2007.

40. Robe PA, Bentires-Alj M, Bonif M, Rogister B, Deprez M, Haddada H, Khac MT, Jolois O, Erkmen K, Merville MP, et al: In vitro and in vivo activity of the nuclear factor-kappaB inhibitor sulfasalazine in human glioblastomas. Clin Cancer Res 10: 5595-5603, 2004.

41. Abdel-Latif MMM, O'Riordan J, Windle HJ, Carton E, Ravi N, Kelleher D and Reynolds JV: NF-kappaB activation in esophageal adenocarcinoma: Relationship to Barrett's metaplasia, survival, and response to neoadjuvant chemoradiotherapy. Ann Surg 239: 491-500, 2004.

42. Abdel Latif MM, O'Riordan JN, Kelleher D and Reynolds JV: Activated nuclear factor-kappaB and cytokine profiles in the esophagus parallel tumor regression following neoadjuvant chemoradiotherapy. Dis Esophagus 18: 246-252, 2005.

43. Konturek PC, Nikiforuk A, Kania J, Raithel M, Hahn EG and Mühldorfer S: Activation of NFkappaB represents the central event in the neoplastic progression associated with Barrett's esophagus: A possible link to the inflammation and overexpression of COX-2, PPARgamma and growth factors. Dig Dis Sci 49: 1075-1083, 2004

44. Izzo JG, Correa AM, Wu TT, Malhotra U, Chao CK, Luthra R, Ensor J, Dekovich A, Liao Z, Hittelman WN, et al: Pretherapy nuclear factor-kappaB status, chemoradiation resistance, and metastatic progression in esophageal carcinoma. Mol Cancer Ther 5: 2844-2850, 2006.

45. Izzo JG, Malhotra U, Wu TT, Ensor J, Luthra R, Lee JH, Swisher SG, Liao Z, Chao KS, Hittelman WN, et al: Association of activated transcription factor nuclear factor kappab with chemoradiation resistance and poor outcome in esophageal carcinoma. J Clin Oncol 24: 748-754, 2006.

46. Izzo JG, Malhotra U, Wu TT, Luthra R, Correa AM, Swisher SG, Hofstetter W, Chao KS, Hung MC and Ajani JA: Clinical biology of esophageal adenocarcinoma after surgery is influenced by nuclear factor-kappaB expression. Cancer Epidemiol Biomarkers Prev 16: 1200-1205, 2007. 